\title{
Früherkennung der Alzheimer-Krankheit mit Biomarkern der Bildgebung oder aus dem Liquor
}

Timo Grimmer

Zentrum für kognitive Störungen, Klinik und Poliklinik für Psychiatrie und Psychotherapie, Klinikum rechts der Isar, Technische Universität München

\section{ZUSAMMENFASSUNG}

Seit kurzem ist es möglich, die Alzheimer-Krankheit als die häufigste Ursache demenzieller Syndrome anhand von Biomarkern unabhängig von der klinischen Symptomatik zu diagnostizieren. Gerade die Biomarker, die die Amyloid-Pathologie anzeigen, werden bereits Jahre vor Auftreten der ersten klinischen Symptome positiv; werden zusätzlich die Biomarker der Tau-Pa- thologie und der Neurodegeneration positiv, erhöht sich die diagnostische Spezifität und die Wahrscheinlichkeit klinischer Verschlechterung im kürzeren Verlauf. Es wird die Güte der Biomarker für die Früherkennung der Alzheimer-Krankheit und für die Prognose des klinischen Verlaufs dargestellt.

Die Alzheimer-Biomarker erlauben die Früherkennung der Alzheimer-Krankheit, bevor sich ein demenzielles Syndrom entwickelt. Damit können Patienten identifiziert werden, bei denen Interventionen zur Verzögerung des Fortschreitens der klinischen Symptomatik und des Verhinderns der Demenz erprobt werden.

\section{Einleitung}

Im Jahr 2011 wurden in den Forschungskriterien der Alzheimer-Krankheit Biomarker im Stadium der Demenz, also bei nachgelassener Kognition in mehreren Domänen und darauf resultierender Beeinträchtigung in der selbstständigen Alltagsbewältigung [1], in der prädemenziellen Phase der leichten kognitiven Störung [2], bei also noch weitgehend erhaltener selbstständiger Alltagsbewältigung, sowie in der asymptomatischen Phase [3], also ohne objektivierbare kognitive Defizite, aufgenommen. 2018 wurde in den aktualisierten Kriterien die Diagnose der Alzheimer-Krankheit, unabhängig von klinischen Stadien, allein auf Basis der Biomarker als Zeichen der pathologischen Veränderungen möglich [4]. Angelehnt an das Klassifikationssystem der Tumorerkrankungen kann ein Mensch mit Alzheimer-Krankheit auf dem AT(N)-System abgebildet werden. Basierend auf den typischen pathologischen Veränderungen der Alzheimer-Krankheit - den überwiegend aus dem Peptid Amyloid bestehenden Alzheimer-Plaques, den von hyperphosphoryliertem Tau geprägten Neurofibrillen sowie der neuronalen Schädigung mit Synapsen und Neuronenverlust - wird die Abnormalität eines Biomarkers für Amyloid als A+, eines Markers für die Tau-Pathologie als T+ und eines Markers für Neurodegeneration als $(N)+$ bezeichnet. Für die Diagnose einer Alzheimer-Krankheit bedarf es, wie in den pathologischen Kriterien auch, eines A+T+-Stadiums, die Neurodegeneration ist fakultativ, und die entsprechenden Biomarker werden als unspezifisch für die Alzheimer-Krankheit angesehen, daher wird das $\mathrm{N}$ als $(\mathrm{N})$ angegeben.

Die Biomarker der Alzheimer-Krankheit umfassen gegenwärtig sowohl bildgebende als auch Liquormarker. Auf deren Güte zur Früherkennung der Alzheimer-Krankheit soll anhand exemplarischer jüngerer Studien in diesem Beitrag eingegangen werden. Die jüngsten Entwicklungen zu Biomarkern im Blut werden hier nicht behandelt.

\section{Entwicklung der Biomarker-} Abnormalitäten im Laufe der Alzheimer-Krankheit

Daten aus der Beobachtung von Menschen mit einer erblichen Form der Alzheimer-Krankheit mit autosomal-dominantem Erbgang im Rahmen des DIAN-Konsortiums ( $\triangleright$ Abb. 1) [5] als auch Daten von Menschen mit Alzheimer-Krankheit ohne nachgewiesene erbliche Genese, („sporadische“ Alzheimer-Krankheit) aus dem australischen Konsortium AIBL ( A Abb. 2) [6], zeigten, dass im Rahmen des Fortschreitens der Erkrankung zunächst die Biomarker für Amyloid positiv werden; in der Regel bereits viele Jahre vor dem Auftreten erster klinischer Symptome wie Vergesslichkeit. Im Weiteren werden die Biomarker der Tau-Pathologie positiv, meist mit Beginn der klinischen Symptomatik, und im Folgenden die Biomarker der Neurodegeneration. Diese zeitliche Reihenfolge der Biomarker-Abnormalitäten passt gut zur Amyloid-Kaskaden-Hypothese der Alzheimer-Krankheit [7].

\section{Amyloid-Pathologieanzeigende Biomarker}

Diese umfassen gegenwärtig den Nachweis von Amyloid-Ablagerungen im Gehirn mithilfe spezifischer Radiopharmaka in der Positronenemissionstomografie (PET) und den Nachweis der verminderten Konzentration des Amyloid-Fragments 1-42 im Liquor cerebrospinales, mög- 


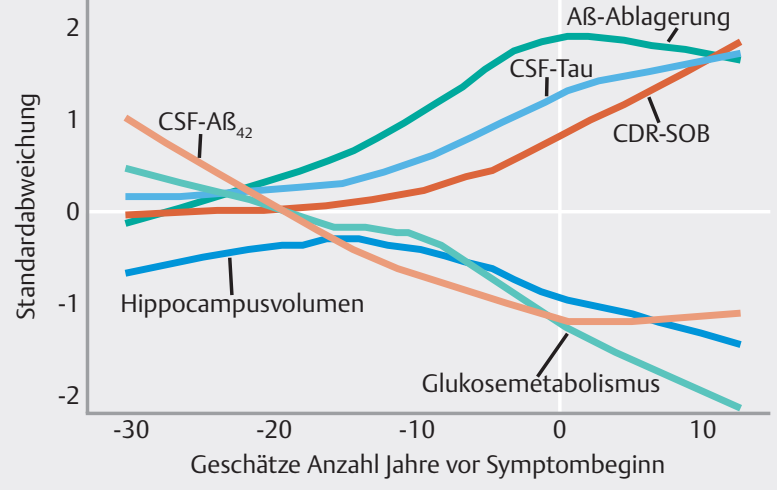

- Abb. 1 Veränderung der Biomarker bei autosomal-dominanter Alzheimer-Krankheit (Quelle: modif. nach [5])

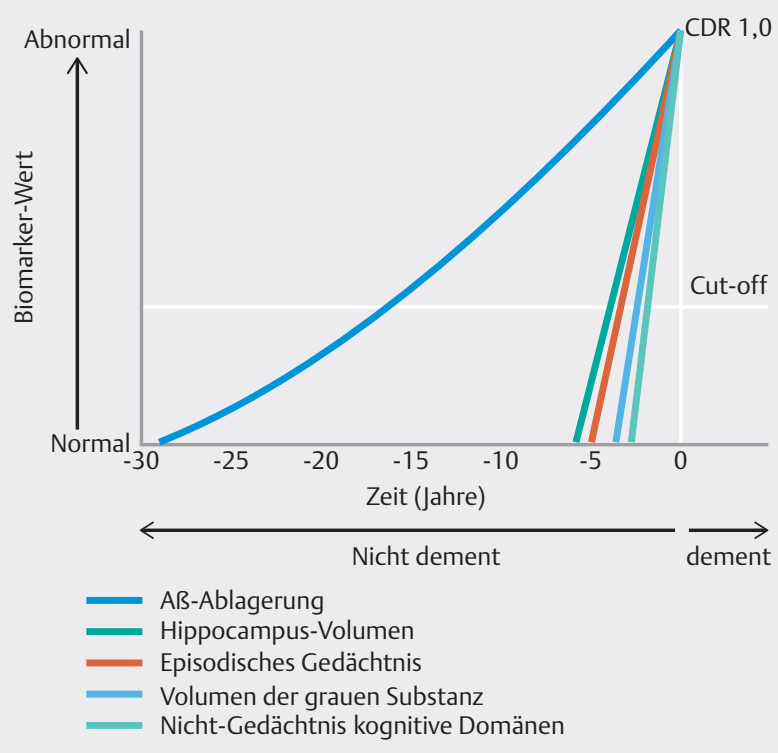

- Abb. 2 Veränderung der Biomarker bei sporadischer Alzheimer-Krankheit (Quelle: modif. nach [6])

licherweise noch genauer des verminderten Quotienten Amyloid1-42/1-40 im Liquor.

Amyloid-PET ist ein quantitativer Marker, der sehr eng mit der Dichte der histopathologisch gezählten Amyloid-Plaques korreliert [8, 9], und ist seit einigen Jahren als zugelassenes Diagnostikum mit kommerziellen Markern verfügbar. Es ist ein äußerst zuverlässiger Marker der Frühdiagnose der Alzheimer-Krankheit, der, je nach angelegtem Grenzwert, bei erblichen Formen 5-10 Jahre vor Symptombeginn [5] und bei sporadischen Formen bis zu 15 Jahre vor Eintritt des demenziellen Syndroms [10] positiv wird. Zusätzlich ist er ein hilfreicher Marker der Differenzialdiagnose, der nach ersten Forschungen 2008 [11] seit einigen Jahren allgemein zur Abgrenzung von Ursachen demenzieller Syndrome bei denen Amyloid ein Kenn- zeichen ist, von solchen, bei denen das nicht der Fall ist [12], etabliert ist. Bei Menschen mit leichter kognitiver Störung (mild cognitive impairment, $\mathrm{MCl}$ ), einem ätiologisch heterogenen Syndrom, das oft eine prädemenzielle Form der Alzheimer-Krankheit darstellt, ist eine Amyloid-PET häufig positiv ( $\$$ Abb. 3) [13]. Versucht man bei Menschen mit dem Syndrom der leichten kognitiven Störung eine Vorhersage des klinischen Verlaufs nach 2 Jahren unter Zuhilfenahme einer Amyloid-PET, so schließt ein negatives PET die Entwicklung einer Demenz bei zugrunde liegender Alzheimer-Krankheit aus (negativer prädiktiver Wert, NPV $=100 \%$ ), natürlich nicht das Voranschreiten zu einer Demenz aufgrund einer nicht durch Amyloid verursachter Ätiologie. Ein positives Amyloid-PET hingegen schließt eine Remission der kognitiven Störungen im Verlauf aus, nach 2 Jahren kann der Betroffene aber weiterhin an einer leichten kognitiven Störungen bei Alzheimer-Krankheit leiden oder bereits eine Demenz bei Alzheimer-Krankheit entwickelt haben $[14,15]$.

Die S3-Leitlinie Demenzen empfiehlt die Amyloid-PET gegenwärtig als ein mögliches Diagnostikum zur Differenzialdiagnose unklarer demenzieller Entwicklungen [16]. Möglicherwiese wird sich der Stellenwert künftig erhöhen. Eine Studie an über 6900 Patienten mit leichter kognitiver Störung und an über 4500 Patienten mit Demenz zeigte, dass das Ergebnis einer Amyloid-PET in über $60 \%$ der Fälle zu einer Änderung des klinischen Managements, meistens in Form einer Anpassung der Medikation, führte [17].

Der erniedrigte Spiegel von $\beta$-Amyloid 1-42 in Liquor wird, mit bis zu 10 Jahren vor Symptombeginn, ähnlich frühzeitig im Krankheitsverlauf auffällig wie der Biomarker Amyloid-PET, wie zumindest bei den autosomal-dominanten Formen der Alzheimer-Krankheit gezeigt wurde ( $\mathbf{A b b}$. 1) [5]. Möglicherweise ist der Quotient $\beta$-Amyloid 1-42/140 noch genauer für die Erkennung der Alzheimer-Krankheit [18]. Menschen mit leichter kognitiver Störung haben bei Vorliegen eines positiven Biomarkers von $\beta$-Amyloid 1-42 in Liquor ein erhöhtes Risiko kognitiver Verschlechterung in den kommenden 5 Jahren; insbesondere bei gleichzeitiger Positivität für einen Biomarker der Neurodegeneration (hier: FDG-PET) steigt das Risiko auf eine Hazard ratio (HR) von 3,36 bis 3,85 an [19].

Bei der Frage, ob man den Biomarker für Amyloid besser mit PET oder mit Liquor bestimmen soll, sollten neben den Erwägungen der Verfügbarkeit, der Kosten, der Kostenerstattung, der Strahlenexposition und der Invasivität, auch bedacht werden, dass die beiden Verfahren mit AUCs von ca. 85 \% für die Diagnose einer zugrunde liegenden Alzheimer-Krankheit wahrscheinlich gleichwertig sind [20], Amyloid-PET aber ein quantifizierbarer Marker der kumulativen Amyloid-Pathologie ist und $\beta$-Amyloid 1-42 im Liquor eher der verminderten Amyloid-Clearance, und dass das Ausmaß der Auffälligkeiten in beiden Biomarkern entsprechend nur in liquornahen Hirnarealen korreliert [21]. 


\section{Tau-Pathologieanzeigende Biomarker}

Diese beinhalten gegenwärtig den Nachweis von Tau-Ablagerungen im Gehirn mithilfe spezifischer Radiopharmaka in der PET als auch den Nachweis erhöhter phosphorylierter Tau-Peptide, typischerweise des Tau181, im Liquor. Die Tau-anzeigenden Biomarker werden typischerweise erst später als die Amyloid-anzeigenden Biomarker im Verlauf der Alzheimer-Krankheit positiv. Zur frühen Diagnose einer Alzheimer-Krankheit eignen sich diese trotz der entsprechenden geringeren Sensitivität durch ihre hohe Spezifität, sodass bspw. mit Tau-PET eine Unterscheidung zwischen Gesunden und Patienten mit leichter kognitiver Störung bei zugrundel iegender Alzheimer-Krankheit immer noch mit einer AUC von $86 \%$ gelingt [22].

\section{Neurodegenerationanzeigende Biomarker}

Diese eher heterogene Kategorie beinhaltet die Erhöhung aller durch Pan-Tau-Antikörper gemessene Tau-Peptide im Liquor, das total Tau (tTau), weiterhin den Alzheimer-typischen Hypometabolismus in der FDG-PET, als auch das verminderte Hirnvolumen, gemessen über voxelbasierte Morphometrie. Diese Biomarker stellen letztlich eine sehr heterogene Gruppe dar, und Patienten mit Alzheimer-Krankheit sind je nach Stadium nicht auf jedem der Marker positiv [23]. Im Verlauf der Alzheimer-Krankheit werden diese erst nach Symptombeginn, (> Abb. 1) [5], und wenige Jahre vor der Entwicklung eines demenziellen Syndroms ( $\triangleright$ Abb. 2) [6], also relativ spät, positiv. Herausgehoben unter diesen Biomarkern soll hier einmal die strukturelle Hirnatrophie, gemessen mit MRT, werden. Ein zerebrales MRT wird ohnehin regelhaft zur Diagnostik eines demenziellen Syndroms durchgeführt („Soll“-Empfehlung, Empfehlungsgrad A [16]). Während die visuelle Beurteilung der Atrophie seit vielen Jahren etabliert ist [24], könnte der Nutzen als ein Biomarker für die Alzheimer-Krankheit über die Verwendung kommerziell verfügbarer und schnell durchführbarer automatisierter Verfahren zur objektiven Bestimmung der Atrophie zusätzlich steigen. So gelang unlängst eine Unterscheidung zwischen Gesunden und Menschen mit leichter kognitiver Störung bei Alzheimer-Krankheit unter zusätzlicher Verwendung solcher Auswertemethodik mit einer AUC von bis zu $90 \%$ [25].

\section{Fortschritte in der liquorbasierten Biomarker-Diagnostik}

Während die Vergleichbarkeit der im Liquor bestimmten Biomarker der Alzheimer-Krankheit zwischen Tests und Laboren über viele Jahre eher schwierig war, haben sich in den letzten Jahren durch die Verbreitung von automatisierten Analyseplattformen und die Verbesserung der Qualität der Tests in der Zwischenzeit Variationskoeffizienten von, je nach Parameter, höchstens $10 \%$ ergeben (https://neurophys.gu.se/english/departments/psychiatry_and_neurochemistry/Neurochemical_pathophysiology_and_diagnostics/TheAlzAssQCProgram/about).
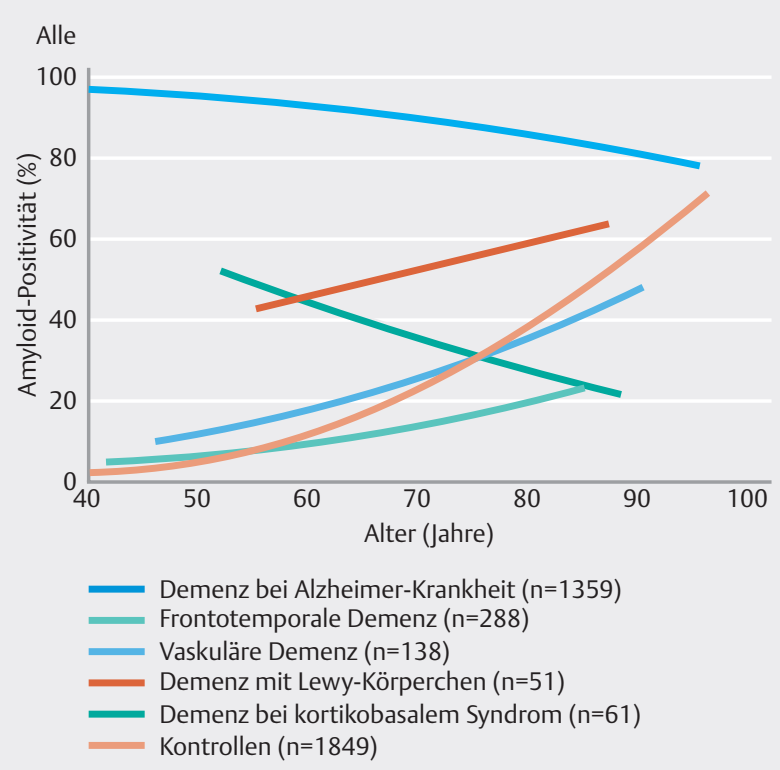

- Abb. 3 Amyloid-Positivität bei unterschiedlichen neurodegenerativen Erkrankungen (Quelle: modif. nach [13])

\section{FAZIT FÜR DIE PRAXIS}

Seit einigen Jahren ist es möglich, die Alzheimer-Krankheit unabhängig von den klinischen Symptomen mittels Biomarker zu diagnostizieren. Während Amyloid-Biomarker als erste im Krankheitsverlauf positiv werden, erhöhen die Biomarker der Tau-Pathologie und der Neurodegeneration die Spezifität der Diagnose. Biomarker für die Bildgebung mit PET als auch für die Diagnostik mit Liquor stehen kommerziell zur Verfügung und erlauben eine diagnostische Trennung der Alzheimer-Krankheit von anderen Ursachen kognitiver Beeinträchtigung und Demenz. Die Alzheimer-Biomarker erlauben die Früherkennung der Alzheimer-Krankheit bereits Jahre bevor sich ein demenzielles Syndrom entwickelt. Damit können auch Patienten identifiziert werden, bei denen Interventionen zur Verzögerung des Fortschreitens der klinischen Symptomatik und des Verhinderns der Demenz erprobt werden.

Wissenschaftlich verantwortlich gemäß Zertifizierungsbedingungen für diesen Beitrag

PD Dr. med. Timo Grimmer, München. 


\section{Interessenkonflikt}

Erklärung zu finanziellen Interessen

Forschungsförderung erhalten: ja. Honorare/geldwerten Vorteil für Referententätigkeit erhalten: ja; Bezahlter Berater/interner Schlungsreferent/Gehaltsempfänger: ja; Patent/Geschäftsanteile/Aktien (Autor/Partner, Ehepartner, Kinder) an Firma: nein

Erklärung zu nicht finanziellen Interessen

Es liegen keine Interessenkonflikte vor.

\section{Korrespondenzadresse}

\section{PD Dr. Timo Grimmer}

Zentrum für kognitive Störungen, Klinik und Poliklinik für Psychiatrie und Psychotherapie

Klinikum rechts der Isar der Technischen Universität München

Ismaninger Str. 22, 81675 München

Tel. 089/41404262, Fax 089/41404923

t.grimmer@tum.de

\section{Literatur}

[1] McKhann GM, Knopman DS, Chertkow $\mathrm{H}$ et al. The diagnosis of dementia due to Alzheimer's disease: recommendations from the National Institute on Aging-Alzheimer's Association workgroups on diagnostic guidelines for Alzheimer's disease. Alzheimers Dement 2011; 7: 263-269

[2] Albert MS, DeKosky ST, Dickson D et al. The diagnosis of mild cognitive impairment due to Alzheimer's disease: recommendations from the National Institute on Aging-Alzheimer's Association workgroups on diagnostic guidelines for Alzheimer's disease. Alzheimers Dement 2011; 7: 270-279

[3] Sperling RA, Aisen PS, et al. Toward defining the preclinical stages of Alzheimer's disease: recommendations from the National Institute on Aging-Alzheimer's Association workgroups on diagnostic guidelines for Alzheimer's disease. Alzheimers Dement 2011; 7: 280-292

[4] Jack CR, Bennett DA, Blennow K et al. NIA-AA Research Framework: Toward a biological definition of Alzheimer's disease. Alzheimers Dement 2018; 14: 535-562

[5] Bateman RJ, Xiong C, Benzinger TL et al. Clinical and biomarker changes in dominantly inherited Alzheimer's disease. N Engl J Med 2012; 367: 795-804

[6] Villemagne VL, Burnham S, Bourgeat $P$ et al. Amyloid beta deposition, neurodegeneration, and cognitive decline in sporadic Alzheimer's disease: a prospective cohort study. Lancet Neurol 2013; 12: 357-367

[7] Hardy J, Selkoe DJ. The amyloid hypothesis of Alzheimer's disease: progress and problems on the road to therapeutics. Science 2002; 297: 353-356

[8] Ikonomovic MD, Klunk WE, Abrahamson EE et al. Post-mortem correlates of in vivo PiB-PET amyloid imaging in a typical case of Alzheimer's disease. Brain 2008; 131: 1630-1645

[9] Clark CM, Pontecorvo M], Beach TG et al. Cerebral PET with florbetapir compared with neuropathology at autopsy for detection of neuritic amyloid-beta plaques: a prospective cohort study. Lancet Neurol 2012; 11: 669-678

[10] Frost $\mathrm{S}$, Kanagasingam $\mathrm{Y}$, Sohrabi $\mathrm{H}$ et al. Retinal vascular biomarkers for early detection and monitoring of Alzheimer's disease. Transl Psychiatry 2013; 3: e233
[11] Drzezga A, Grimmer T, Henriksen G et al. Imaging of Amyloid Plaques and Cerebral Glucose Metabolism in Semantic Dementia and Alzheimer's Disease. Neuroimage 2008; 39: 619-633

[12] Ossenkoppele R, Jansen W], Rabinovici GD et al. Prevalence of amyloid PET positivity in dementia syndromes: a meta-analysis. Jama 2015; 313: 1939-1949

[13] Jansen WJ, Ossenkoppele R, Knol DL et al. Prevalence of cerebral amyloid pathology in persons without dementia: a meta-analysis. Jama 2015; 313: 1924-1938

[14] Grimmer T, Wutz C, Drzezga A et al. The usefulness of amyloid imaging in predicting the clinical outcome after two years in subjects with mild cognitive impairment. Curr Alzheimer Res 2013; 10: 82-85

[15] Grimmer T, Wutz C, Alexopoulos P et al. Visual Versus Fully Automated Analyses of 18F-FDG and Amyloid PET for Prediction of Dementia Due to Alzheimer Disease in Mild Cognitive Impairment. J Nucl Med 2016; 57: 204-207

[16] Deutsche Gesellschaft für Psychiatrie und Psychotherapie,PuND, Deutsche Gesellschaft für Neurologie (DGN). S3-Leitlinie „Demenzen“. Berlin 2016.

[17] Rabinovici GD, Gatsonis C, Apgar C et al. Association of Amyloid Positron Emission Tomography With Subsequent Change in Clinical Management Among Medicare Beneficiaries With Mild Cognitive Impairment or Dementia. JAMA 2019; 321: 1286-1294

[18] Pannee J, Portelius E, Minthon L et al. Reference measurement procedure for CSF amyloid beta (Abeta)1-42 and the CSF Abeta1-42/Abeta1-40 ratio - a cross-validation study against amyloid PET. J Neurochem 2016; 139: 651-658

[19] Caroli A, Prestia A, Galluzzi S et al. Mild cognitive impairment with suspected nonamyloid pathology (SNAP): Prediction of progression. Neurology 2015; 84: 508-515

[20] Mattsson N, Insel PS, Landau S et al. Diagnostic accuracy of CSF Ab42 and florbetapir PET for Alzheimer's disease. Ann Clin Transl Neurol 2014; 1: 534-543

[21] Grimmer T, Riemenschneider M, Forstl $\mathrm{H}$ et al. Beta amyloid in Alzheimer's disease: increased deposition in brain is reflected in reduced concentration in cerebrospinal fluid. Biol Psychiatry 2009; 65: 927-934

[22] Ossenkoppele R, Rabinovici GD, Smith R et al. Discriminative Accuracy of [18 F]flortaucipir Positron Emission Tomography for Alzheimer Disease vs Other Neurodegenerative Disorders. JAMA 2018; 320: 1151-1162

[23] Alexopoulos P, Kriett L, Haller B et al. Limited agreement between biomarkers of neuronal injury at different stages of Alzheimer's disease. Alzheimers dement 2014; 10: 684-689

[24] Scheltens P, Leys D, Barkhof F et al. Atrophy of medial temporal lobes on MRI in "probable" Alzheimer's disease and normal ageing: diagnostic value and neuropsychological correlates. J Neurol Neurosurg Psychiatry 1992; 55: 967-972

[25] Hedderich DM, Spiro JE, Goldhardt O et al. Increasing Diagnostic Accuracy of Mild Cognitive Impairment due to Alzheimer's Disease by User-Independent, Web-Based Whole-Brain Volumetry. J Alzheimers Dis 2018; 65: 1459-1467

Bibliografie

DOI https://doi.org/10.1055/a-0949-1367

Nervenheilkunde 2019; 38: 813-816

(c) Georg Thieme Verlag KG Stuttgart · New York ISSN 0722-1541 


\section{Punkte sammeln auf CM/F.thieme.de}

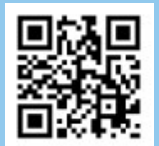

Diese Fortbildungseinheit ist in der Regel 12 Monate online für die Teilnahme verfügbar. Den genauen Einsendeschluss finden Sie unter https://eref.thieme.de/CXDDIJY.

Sollten Sie Fragen zur Online-Teilnahme haben, finden Sie unter https://cme.thieme.de/hilfe eine ausführliche Anleitung. Wir wünschen viel Erfolg beim Beantworten der Fragen!

Unter https://eref.thieme.de/CXDDIJY oder über den QR-Code kommen Sie direkt zur Startseite des Wissenstests.

VNR 2760512019156640367

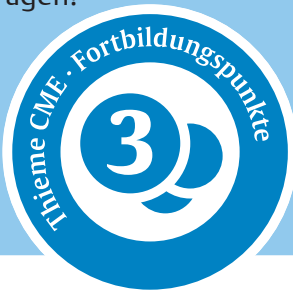

\section{Frage 1}

Welche Aussage trifft zu? Alzheimer-Krankheit ...

A und Demenz sind Synonyme.

B kann nur klinisch diagnostiziert werden.

C kann zu Lebzeiten mit Hilfe von Biomarkern diagnostiziert werden.

D und Altersdemenz sind Synonyme.

E kann nur im Stadium der Demenz diagnostiziert werden.

\section{Frage 2}

Welche Aussage trifft nicht zu? Etablierte Biomarker zur Diagnose der Alzheimer-Krankheit im Liquor sind ...
A beta-Amyloid 1-42.
B Quotient beta-Amyloid 1-42/beta-Amyloid 1-40.
C Neurofilament Leichtketten (NfL).
D Phospho-tau 181.
E Gesamt-tau (tTau).

\section{Frage 3}

Welche Aussage trifft zu? Das AT(N)-System ...

A wird zur Charakterisierung von Tumoren verwendet.

B erlaubt eine biomarkerbasierte Diagnose der AlzheimerKrankheit.

C bildet den Schwergrad eines demenziellen Syndroms ab.

D darf nur im Stadium der Demenz angewendet werden.

$\mathrm{E}$ ist in der ICD-10 hinterlegt.

\section{Frage 4}

Welche Aussage trifft nicht zu?

A Biomarker der Neurodegeneration sind im Verlauf der Alzheimer-Krankheit typischerweise zuerst auffällig.

B Der Nachweis eines auffälligen Biomarkers für Amyloid ist nicht ausreichend zur Diagnose einer Alzheimer-Krankheit nach dem AT(N)-System.

C Der Nachweis eines auffälligen Biomarkers für Tau ist nicht ausreichend zur Diagnose einer Alzheimer-Krankheit nach $\operatorname{dem} \mathrm{AT}(\mathrm{N})$-System.

D Der Nachweis eines auffälligen Biomarkers für Neurodegeneration ist nicht ausreichend zur Diagnose einer AlzheimerKrankheit nach dem AT(N)-System.

E Die Amyloid-Biomarker können bei Menschen mit AlzheimerKrankheit bereits Jahre vor den ersten Symptomen auffällig sein.

\section{Frage 5}

Welche Aussage trifft zu? Mit Hilfe der Biomarker der AlzheimerKrankheit kann diese ...

A unabhängig vom klinischen Stadium diagnostiziert werden.

B im asymptomatischen Stadium nicht diagnostiziert werden.

C im Stadium der leichten kognitiven Störung nicht diagnostiziert werden.

D im Stadium der beginnenden Demenz nicht diagnostiziert werden.

E im Stadium der fortgeschrittenen Demenz nicht diagnostiziert werden.

\section{Frage 6}

Welche Aussage trifft nicht zu?

A Amyloid-PET ist ein Marker für A nach dem AT(N)-System.

B $\beta$-Amyloid 1-42 im Liquor ist ein Marker für $A$ nach dem AT(N)-System.

C Gesamt-tau (tTau) im Liquor ist ein Marker für T nach dem AT(N)-System.

D Phospho-tau 181 (pTau181) im Liquor ist ein Marker für T nach dem AT(N)-System.

E Tau-PET ist ein Marker für T nach dem AT(N)-System.

- Weitere Fragen auf der folgenden Seite ... 


\section{Punkte sammeln auf CME.thieme.de}

Fortsetzung ...

\section{Frage 7}

Welche Aussage trifft zu?

A Amyloid-Abnormalitäten können nur durch die Bestimmung von $\beta$-Amyloid 1-42 im Liquor nachgewiesen werden.

B Tau-Abnormalitäten können nur durch die Durchführung eines Tau-PET nachgewiesen werden.

C Die Durchführung eines Amyloid-PET wird nach S3-Leitlinie nicht empfohlen.

D Für die Diagnose einer Amyloid-Abnormalität (A + nach AT(N)) sind Amyloid-PET und $\beta$-Amyloid 1-42 im Liquor vergleichbar gute Biomarker.

E Amyloid-PET und $\beta$-Amyloid 1-42 im Liquor sind gleichwertig hinsichtlich der Quantifizierbarkeit zerebraler AmyloidAblagerungen.

\section{Frage 8}

Welche Aussage hinsichtlich der Biomarker der Neurodegeneration nach dem AT(N)-System trifft nicht zu?

A Die Hippocampusvolumetrie im MRT ist ein Marker für(N) nach dem AT(N)-System.

B Gesamt-tau (tTau) im Liquor ist ein Marker für (N) nach dem AT(N)-System.

C FDG-PET ist ein Marker für (N) nach dem AT(N)-System.

D Ein Patient, der auf einem Marker für $(\mathrm{N})$ positiv ist, ist auf den anderen Markern für $(\mathrm{N})$ ebenfalls positiv.

E Der Nachweis eines auffälligen Biomarkers für Neurodegeneration ist nicht ausreichend zur Diagnose einer AlzheimerKrankheit nach dem AT(N)-System.

\section{Frage 9}

Welche Aussage zu leichter kognitiver Störung (mild cognitive impairment, $\mathrm{MCl}$ ) trifft zu?

A $\mathrm{MCl}$ ist immer durch die Alzheimer-Krankheit bedingt.

B Patienten mit $\mathrm{MCl}$ und positiven Biomarkern der AlzheimerKrankheit entwickeln in den kommenden 2 Jahren zwangsläufig ein demenzielles Syndrom.

C Patienten mit $\mathrm{MCl}$ und negativen Biomarkern der AlzheimerKrankheit entwickeln in den kommenden 2 Jahren in der Regel keine Alzheimer-Demenz.

D Patienten mit $\mathrm{MCl}$ und negativen Biomarkern der AlzheimerKrankheit entwickeln in den kommenden 2 Jahren keinesfalls ein demenzielles Syndrom.

E Bei MCl liegen die Ergebnisse neuropsychologischer Tests in der Regel innerhalb der Alters- und Bildungsnorm.

\section{Frage 10}

Welche Aussage zu Demenz trifft nicht zu?

F Für die Diagnose einer Demenz muss eine Störung wenigstens einer kognitiven Domäne vorliegen.

G Eine Demenz kann nicht allein durch neuropsychologische Tests diagnostiziert werden.

H Die Diagnose einer Demenz erfordert eine Beeinträchtigung in der selbstständigen Alltagsbewältigung.

I Eine Demenz ist nicht immer durch eine Alzheimer-Krankheit bedingt.

」 Eine Demenz kann nicht durch Biomarker diagnostiziert werden. 